\title{
Variational tetrahedral mesh generation from discrete volume data
}

\author{
J. Dardenne - S. Valette - N. Siauve - N. Burais - R. Prost
}

the date of receipt and acceptance should be inserted later

\begin{abstract}
In this paper, we propose a novel tetrahedral mesh generation algorithm, which takes volumic data (voxels) as an input. Our algorithm performs a clustering of the original voxels within a variational framework. A vertex replaces each cluster and the set of created vertices is triangulated in order to obtain a tetrahedral mesh, taking into account both the accuracy of the representation and the elements quality. The resulting meshes exhibit good elements quality with respect to minimal dihedral angle and tetrahedra form factor. Experimental results show that the generated meshes are well suited for Finite Element Simulations.
\end{abstract}

\section{Introduction}

Tetrahedral meshes are widely used in nowadays 3D applications. This is especially true for simulations, where one can evaluate a wide range of different physical phenomena, from electromagnetics to thermics [20] as the determination of temperature distribution in human body submitted to radio-frequency fields is currently impractical using temperature sensors. These simulations are performed on virtual objects. A very commonly used simulation framework is the Finite Elements Method (FEM) which applies to triangular meshes for the $2 \mathrm{D}$ setting, or tetrahedral meshes for the $3 \mathrm{D}$ setting. One caveat of such framework is its need for tetrahedral meshes with good quality, where the quality is generally

J. Dardenne and S. Valette and R. Prost are with:

University of Lyon,

CREATIS-LRMN; CNRS UMR5220; Inserm U630;INSA-Lyon; University of Lyon 1, France.

‘E-mail: name@creatis.insa-lyon.fr

J. Dardenne and N. Siauve and N. Burais are with:

University of Lyon,

AMPERE ; CNRS UMR5005; University of Lyon 1, France.

E-mail: name@univ-lyon1.fr measured in terms of minimal dihedral angle. Indeed, the accuracy and convergence speed of a FEM-based simulation depends on the mesh objective quality. As a consequence, a lot of research was carried out on the topic of mesh generation. The majority of the published algorithms build tetrahedral meshes starting from a previously constructed surface mesh (triangles) which represents the surface of the object to be meshed [21]. The triangular mesh can come from different contexts : Computer Aided Design, 3D laser scanning, and is sometimes modified prior the tetrahedral meshing step, in order to meet certain criteria (triangles quality, as an example).

In this paper, we aim at generating 3D meshes starting from volumic data (voxels). Our motivation stands from the ever-increasing spread of 3D medical imaging facilities which deliver a volumic representation of the human body (or just a part of it). 3D images can be obtained from Magnetic Resonance Imaging (MRI) tomographic scanners or ultrasound imaging. Our algorithm creates a tetrahedral mesh by distributing a user-defined number of vertices in the meshing domain (see figure 1), possibly according to a density map. The vertices distribution is driven by a variational clustering scheme. The vertices laying on the boundary of the object are subject to location constraints in order to keep a good accuracy on the object surface, and stress is put on the quality of the generated triangulation.

Our paper is organized as follows : section 2 is a brief overview of previous tetrahedral meshing algorithms. Section 3 gives some definitions and principles used by our approach (section 4). In section 5, we show some experimental results, where we compare our algorithm with a commercial mesher, both in terms of elements quality and simulation results, and a conclusion follows. 


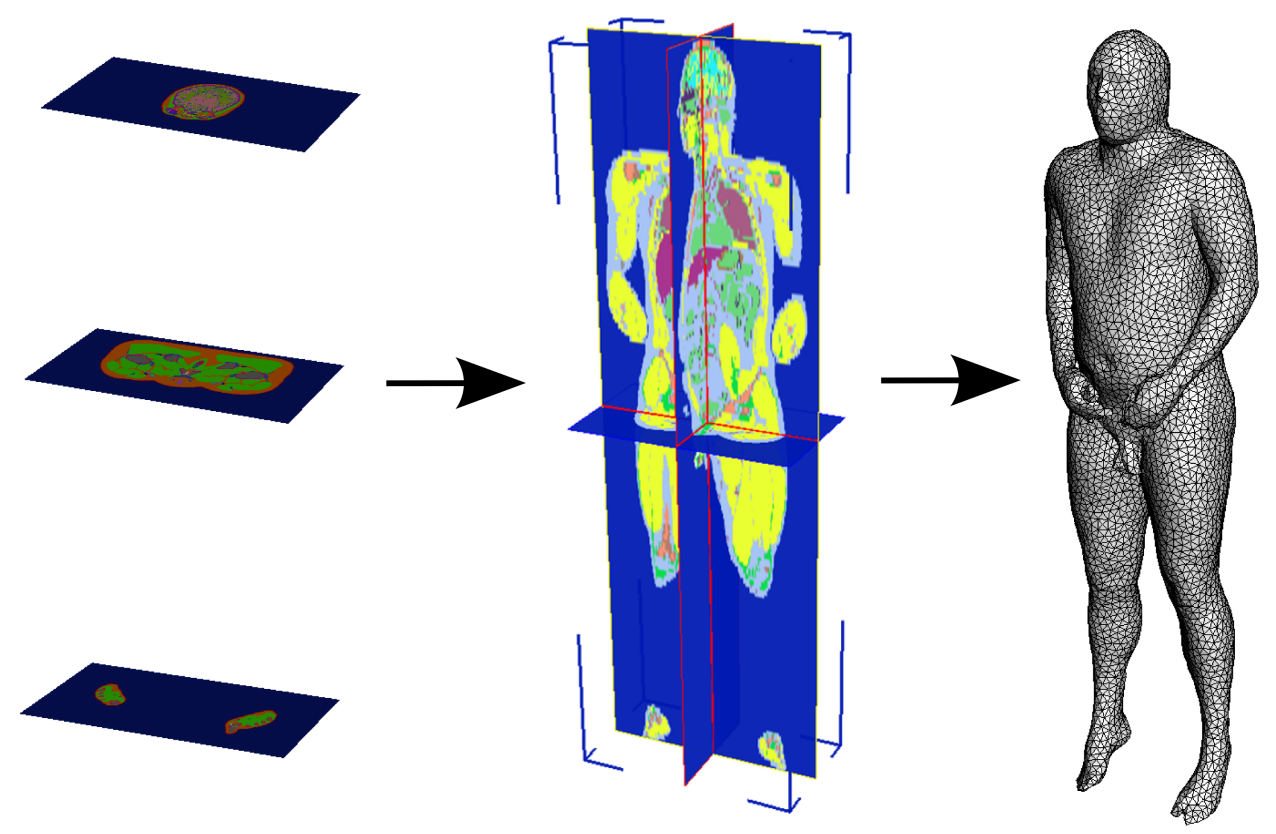

Fig. 1 Meshing the full body in the visible-human-male data-set

\section{Previous works on mesh generation}

Tetrahedral mesh generation has been extensively studied in the last years. We can basically classify existing approaches in three different categories : Delaunay-based approaches, greedy approaches and algorithms relying on a hierarchical decomposition of the space.

\subsection{Delaunay-based methods}

These methods usually try to distribute a set of vertices in the domain to process, and the triangulation is created by constructing the Delaunay triangulation of the vertices. Vertices can be iteratively inserted into the triangulation until the desired density is reached. The main criterion for Delaunay mesh generation is the empty sphere (resp. circle in 2D) criterion : no vertex is located inside the circumscribed spheres (resp. circles) of the mesh tetrahedra (resp. triangles). Unfortunately, when dealing with three dimensions, this criterion is not sufficient to guarantee the quality of the constructed mesh and is very sensitive to truncation errors in practical 3D computations. Sometimes, more than five points are almost located on the same sphere. Degenerated elements called slivers can append, thus penalizing FEM simulations [5]. To solve this problem, [16] proposed an efficient algorithm for arbitrary precision floating-point but inserting new vertices inside the triangulation is sometimes not trivial [18].

\subsection{Greedy approaches}

Greedy approaches generally start from a surface mesh which is filled with tetrahedra by inserting Steiner points inside the mesh and adding new tetrahedra [10,6]. Local criteria drive the points insertion, in order to improve the quality of the generated tetrahedra. Local mesh density near the initial front (i.e. initial boundaries) can be controlled easily. These approaches are fast but the quality of the generated meshes is not the best achievable. Combination of the advantages of the Delaunay and the greedy approaches has been widely used [14]. This approach starts with a Delaunay triangulation of a set of boundary vertices. This is used as a background mesh. New vertices are then added by the advancing front approach. Although the initial Delaunay triangulation is not difficult in 2D, the surface recovery is often troublesome in 3D.

\subsection{Hierarchical decomposition approaches}

These algorithms are based on the decomposition of the domain to be meshed using cubical elements [15]. The elements are recursively subdivided in order to improve the approximation quality of the boundaries or to satisfy a user defined precision. Elements that lie outside the meshing domain, and the elements inside the domain are split into tetrahedra. In [13], a new hierarchical decomposition approach has been proposed, this method fills an isosurface with a mesh and offers theoretical guarantees on dihedral angles between $10.7^{\circ}$ and $164.8^{\circ}$. The drawback of these methods 


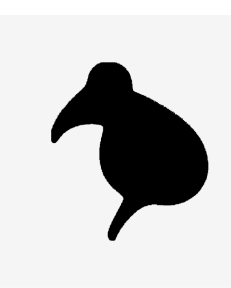

(a)

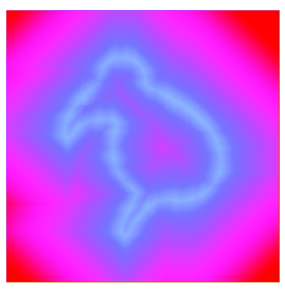

(c) (b)

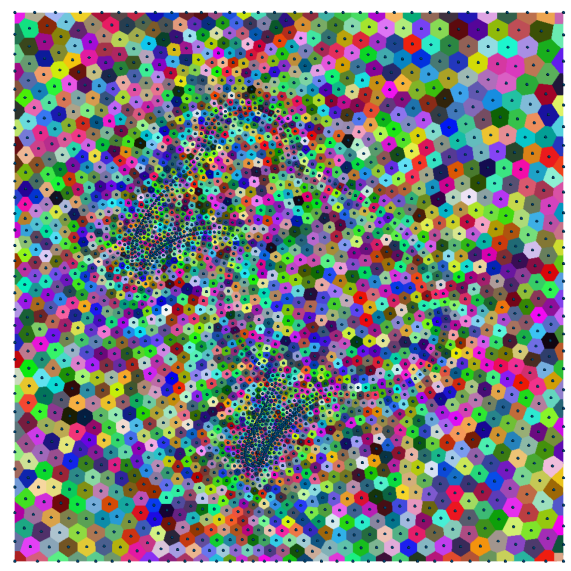

(d)

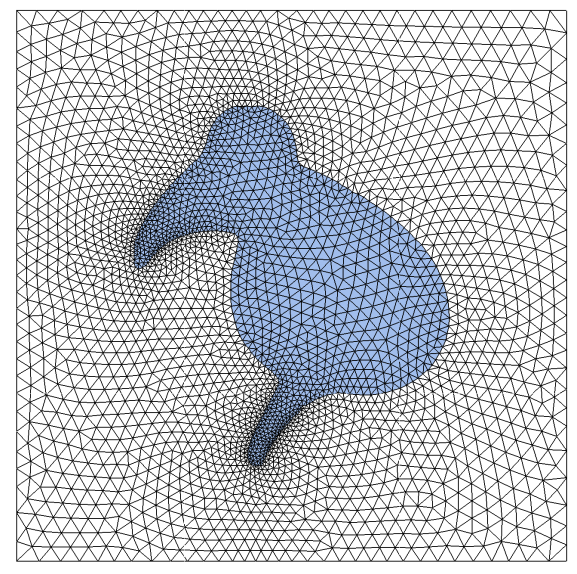

(e)

Fig. 2 2D example : (a) Original image. We compute the medial axis of the shape (b). The medial axis is used to generate a density function (c). Using the density function and the object boundaries, we construct an approximated Centroidal Voronoi Diagram (d) which is finally dualized into the resulting mesh (e).

\subsection{Discussion}

Most of the previously cited approaches share a common point : they take an input surface and enrich it with new vertices to generate the tetrahedra. This can be problematic around the objects boundaries, as the vertices of the input surface can be an important constraint for the resulting mesh, and induce tetrahedra with bad aspect ratio tetrahedra. It has been shown in [19] that the quality of the mesh elements can increase computation time with the Finite Elements Method. A classical approach to increase the mesh elements quality is a post-processing where the slivers are removed [11]. A lot of objective quality criteria exist in the literature [4]. In this paper, we use four quality criteria : The criterion $Q_{1}$ is the minimal dihedral angle $\alpha_{\min }$ of a tetrahedron, which has a maximal value of $\arccos (1 / 3)=70,5^{\circ}$ for a regular tetrahedron. $Q_{2}$ is the maximal dihedral angle $\alpha_{\max }$ of a tetrahedron. The third criterion $Q_{3}$ is the radius ratio. The fourth [19] $Q_{4}$ is defined as:

$Q_{4}=\frac{12}{\bar{l}} \cdot(3 \cdot V)^{2 / 3}$

where $\bar{l}$ is the average edge length of the tetrahedron and $V$ its volume. The criterions $Q_{3}, Q_{4}$ are normalized between 0 and 1 , where 0 denotes a sliver and 1 a regular tetrahedron.

\section{Definitions}

Here we give some definitions used in this paper. We define the entire domain made with voxels as $\Omega . \Omega$ is the union of different objects $O_{i}$ :

$\Omega=\cup_{i} O_{i}$

The outside boundary of $\Omega$ will be noted as $\partial_{\Omega}$. We also define the set of boundaries between the objects as $\partial_{O}$ :

$\partial_{O}=\partial_{\Omega} \cup\left[\cup_{i, j} \partial_{O_{i, j}}\right]$

where $\partial_{O_{i, j}}$ is the boundary between the objects $O_{i}$ and $O_{j}$.

For clarity reasons, we will explain our approach in two dimensions. For a given image (resp. volume) $\Omega$, we associate a graph $G$ so that each pixel (resp. voxel) is associated to a vertex and its neighbour pixels (resp. voxels) are connected with an edge [12]. This graph, the primal graph is denoted $G=(V, E)$. Where $V$ is the set of vertices and $E$ is the set of edges. The dual of the graph $\bar{G}$ represents the inter-pixels (resp. inter-voxels) and edges, as shown by figure 3 .

\section{Our approach}

We propose a novel tetrahedral mesh generation approach with adapted sampling. Our algorithm works directly on the is that they tend to generate bad form factor tetrahedra along the objects boundaries. 


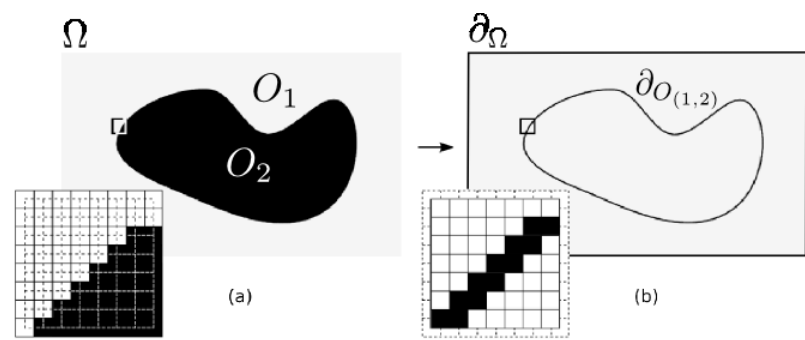

Fig. 3 The primal graph $G$ (left) and its dual $\bar{G}$ (right)

voxels of the input volumes coming from segmented Tomographic Scanners or MRI (illustrated by figure 1). No polygonal input surface is needed. The first step is a clustering of the input data (pixels or voxels) into $n$ cells $C_{i}$, approximating a Centroidal Voronoi Diagram. Each cell $C_{i}$ is given a site $z_{i}$. Such a clustering takes into account a density function $\rho(x)$ and a set of constraints to preserve the boundaries between the objects inside the domain by constraining the sites $z_{i}$ nearby the objects boundaries.

The second step consists in triangulating the sites $z_{i}$, using the dual of the generated clustering. Finally, each constructed tetrahedron is associated to an object $O_{i}$, and we apply a cleaning step to increase the quality of the tetrahedron and to enhance the interface between the different objects.

Figure 2 shows the main steps in our algorithm on a 2D example : the input image is shown in (a). We compute the medial axis of the shape using [7]. With this medial axis, we generate a density map $\rho(x)$ [3] which denotes the local desired vertices density $(c)$. We then construct an approximation of a Centroidal Voronoi Diagram of the input image (d). Finally, the sites of the diagram are triangulated to form the final mesh (e).

\subsection{Domain partition}

The first step of our approach is a partitioning of the input space $\Omega$ by means of clustering, in spirit with Centroidal Voronoi Diagrams (CVD) approaches, where each site $z_{i}$ is also the barycenter of its associated cell:

$z_{i}=\frac{\int_{C_{i}} x \cdot \rho(x) d x}{\int_{C_{i}} \rho(x) d x}$

It is known that CVDs minimize the following energy term [9]:

$E_{V}=\sum_{i=1}^{n}\left(\sum_{p_{j} \in C_{i}} \int_{p_{j}} \rho(x)\left\|x-z_{i}\right\|^{2} d x\right)$

where $x$ is a point inside $\Omega$ and $\rho(x)$ a given density function. In this paper, we minimize $E_{V}$, i.e. we aim at maximizing the cells compacity, as done in [23]. The energy term can be simplified to:

$F=\sum_{i=1}^{n}\left[\sum_{p_{j} \in C_{i}} \rho_{j} z_{i}^{T}\left(z_{i}-2 \gamma_{j}\right)\right]$

where

$\rho_{j}=\int_{p_{j}} \rho(x) d x$

$\gamma_{j}=\frac{1}{\rho_{j}} \int_{p_{j}} x \cdot \rho(x) d x$

where $p_{j}$ is an element of $\bar{G}, \rho_{j}$ is the weight associated to $p_{j}, \gamma_{j}$ is the barycenter of $p_{j}$.

We define the set $B_{i}$ for each region $C_{i}$ as :

$B_{i}=C_{i} \cap \partial_{O}$

Finally, the position of each site $z_{i}$ depends on one condition :

- if $C_{i}$ does not cross any boundary $\left.\left(B_{i}=\varnothing\right)\right)$, the site $z_{i}$ is not constrained. It is set to the cell barycenter: $z_{i}=$ $G_{i}$.

$z_{i}=G_{i}=\frac{\sum_{C_{i}} \rho_{j} \cdot \gamma_{j}}{\sum_{C_{i}} \rho_{j}}$

- If the cell $C_{i}$ crosses the boundary $\partial_{O_{m, n}^{i}}\left(B_{i} \neq \varnothing\right), z_{i}$ is constrained to the barycenter of the boundary:

$$
z_{i}=\frac{\sum_{B_{i}} \rho_{j} \cdot \gamma_{j}}{\sum_{B_{i}} \rho_{j}}
$$

This condition is illustrated by figure 4 . Certain models (like mechanical models) often have boundaries with many distinctive features such as corners and edges that need to be well represented by the mesh. To preserve these features, the site $z_{i}$ is constrained respectively to a point or to the barycenter of the boundary subset. With this approach, we can distribute $N$ cells in the domain $\Omega$ while preserving the boundary between the different objects $\partial_{O}$ and satisfying the desired sampling density $\rho(x)$.

It is often preferable to have variable vertices density in complex and narrow regions. This density is mandatory to have a good local representation of the inter-objects geometry. We proposed in [7], a calculation method for the medial axis from a constrained DVC. The medial axis allows to define the density map [3] in order to mesh more densely certain complex regions of the domain (figure 2.c). Note that this density map can also easily be modified (painted) by the end-user when arbitrary density criteria are needed. For example, we use a density map which in one part possesses uniform weights whereas in the other part respects certain complex regions of the shape (see figure 11). 


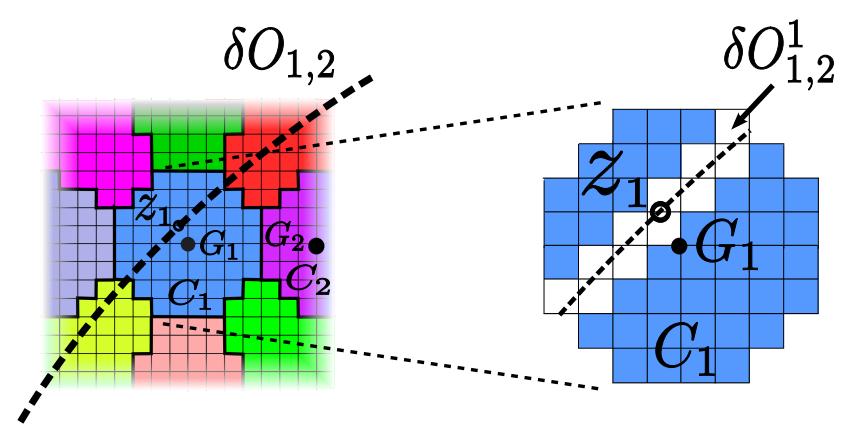

Fig. 4 The cluster $C_{1}$ is constrained, as it crosses the boundary $\partial_{O_{1,2}}$ (equation 11). The location of $z_{1}$ is computed from $\partial_{O_{1,2}^{1}}$ which is the intersection of $C_{1}$ and $\partial_{O_{1,2}}$. The cluster $C_{2}$ is not constrained, as it does not cross any boundary (equation 10 ).

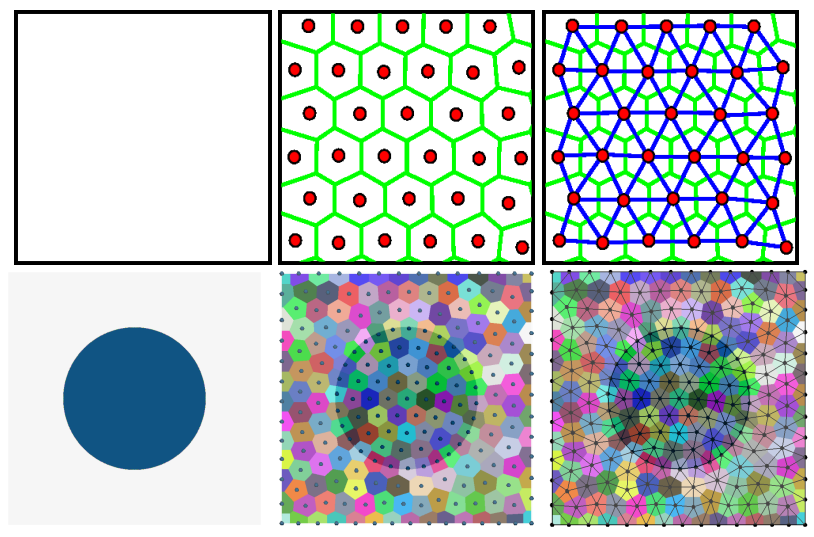

Fig. 5 Left : the input domain $\Omega$. Middle : a set of $n$ points and its Voronoi diagram. Right : the dual triangulation. The top row shows the smooth case (empty box). The bottom row shows the discrete case (circle embedded in a box)

\subsection{Tetrahedral Meshing}

In the general case, a Voronoi Diagram is constructed from a point cloud. It forms a set of cells for which the geometrical dual is the Delaunay triangulation (figure 5). The Voronoi vertices are the center of the triangles circumscribed circle. The Voronoi edges (resp. polygons in 3D) are the mediators of the Delaunay triangulation edges. This definition is in the continuous space case.

In [8], Du and Wang propose to generate meshes that are dual to optimal Voronoi diagrams. In [23], a discrete approximation of the Centroidal Voronoi Diagram was proposed, where the triangulation is built from the dual of this diagram. We use this approach for aggregating together voxels and form afterward the meshing. In analogy with Voronoi diagrams in the smooth case where co-cyclic sites cause ambiguous cases for Delaunay triangulation, cur clustering algorithm generates two kinds of ambiguities:

- when at least four cells form a cycle without inner edge between opposite cells.

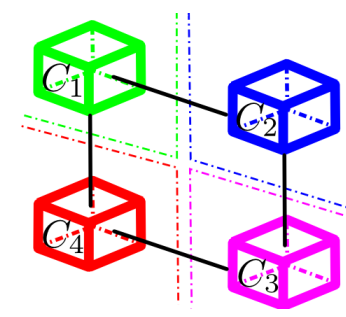

(a)

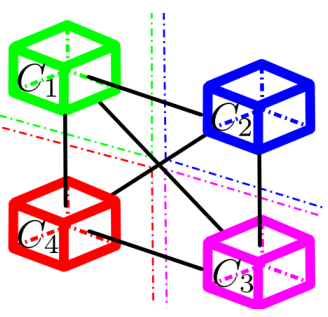

(b)
Fig. 6 Topological ambiguities : (a) four cells form a cycle without inner edge between opposite cells. (b) four cells form a cycle with too many inner edges.

- when at least four cells form a cycle with too many inner edges.

The first ambiguity produces a lack of edges and the second, a surplus (see figure 6). It results a lack or a surplus of tetrahedra in the zones of ambiguities. The relations of adjacency between the Voronoi cells allow to build a set of the acceptable tetrahedra $\Gamma$.

\subsubsection{Propagation}

We use a Greedy algorithm to build the mesh using the tetrahedra of $\Gamma$. Two neighbour tetrahedra are chosen in a region of $\Gamma$ without ambiguity. The front is created with the external faces of both constructed tetrahedra. Then we add new tetrahedra to the front until the front reaches $\partial_{\Omega}$. To resolve the ambiguous cases, we use locally a Constrained Delaunay Triangulation[17].

\subsubsection{Topological Optimization}

We use a set of topological transformations for improving the mesh quality, in spirit with [11]. We do not proceed to insertions or deletions of vertices but we modify the connectivity of these vertices (edge flip in 2D). In three dimensions, these operations can add or delete edges or faces and can modify the number of tetrahedra. The couple $m-n$ denotes that $m$ tetrahedra are removed and $n$ are created, respectively. Figure 1 illustrates several examples, called 2-3 flips, 3-2 flips and 5-4 flips.

- The 3-2 transformation is performed on a configuration where three tetrahedra share the same edge. This transformation can be generalized to more complex configurations, where $m$ tetrahedra share the same edge. In this case, the cycle of tetrahedra around this edge forms a polygon which is triangulated. The faces of this triangulation are associated with the vertices of the initial common edge, to form the new configuration of tetrahedra. This topological transformation deletes one edge and adds one or several faces. 


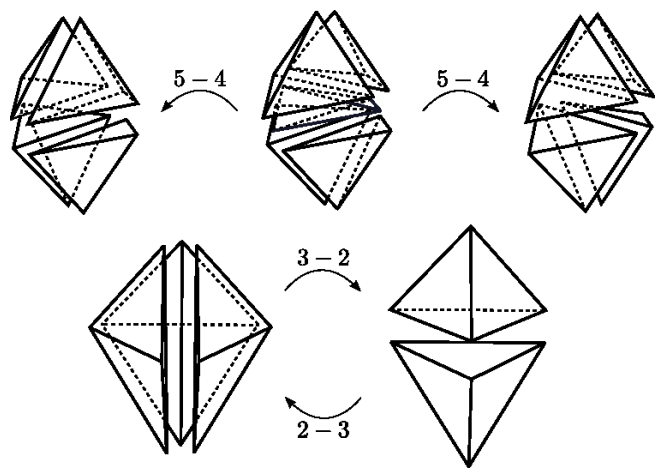

Fig. 7 Examples of topological transformation. Top : 5-4. Bottom : 2-3 and 3-2.

- The 2-3 transformation is the inverse of the transformation 3-2. this transformation can also be widened to its neighborhood. iiiiiii dardenne09.tex The extension consists in the fact that the faces are joined by edges of degree four? to identify a group of suitable faces. ======= The extension consists in the fact that the faces are joined by edges of degree four? to identify a group of suitable faces. $i_{i} i \dot{i} i \dot{i} \quad 1.27$ Note that this topological transformation deletes one or several faces and builds one edge.

- The 5-4 transformation allows to remove slivers. It deletes one sliver and four of its neighbours and creates four new tetrahedra. For a given set of tetrahedra, this transformation can have two different results (see figure 7).

Our optimization algorithm works as follows: A priority queue $Q_{(i d, q u a l)}$ is filled with the set of elements $i d$ (tetrahedra) which quality indicator qual does not meet the desired quality criterion. For each operation, wee pick the element $i d$ having the worst quality and apply the transformation with the best tetrahedra quality improvement. The topological operations are performed until fulfillment of the desired quality criterion or when no new transformation can be performed.

\subsubsection{Boundary Enhancement}

It is necessary to remind that we do not know, a priori, the border of the domain after the clustering. Our knowledge relies only on a set of vertices which belong or not to an object boundary. this can result in spikes on the surface of the objects. To solve this, every tetrahedron is assigned to an object by counting the number if voxels it contains, and to which object they belong. If some voxels belong to two different objects, the tetrahedron is assigned to the dominant class. In such a case, we apply the transformation which best decreases the number of voxels in non-dominant classes. This greatly improves the accuracy of the objects boundaries. An example is illustrated in figure $8:$ the $2 \mathrm{D}$ principle if shown on the top, and a real 3D case is shown at the bottom.

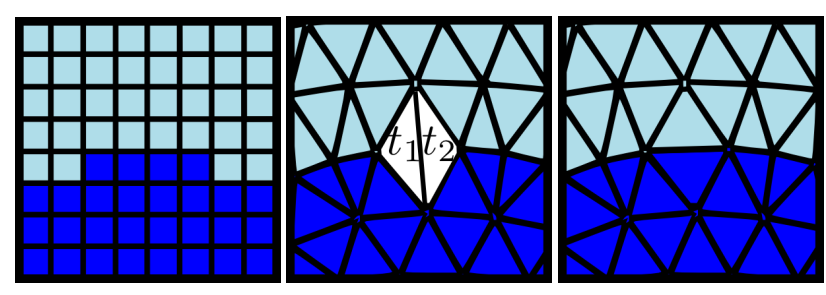

(a)

(b)

(c)

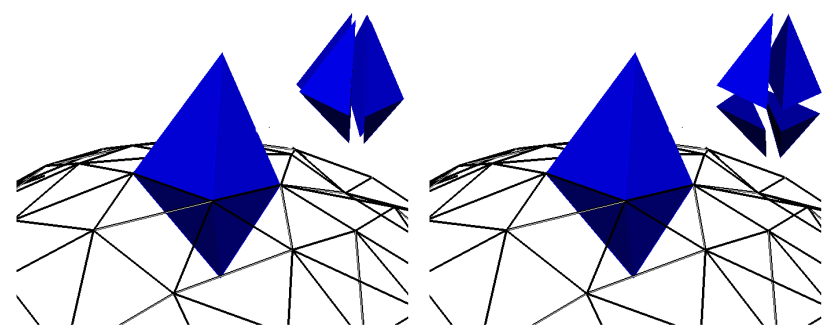

(d)

(e)

Fig. 8 (a) Voxels belonging to two distinct objects. The triangulation (b) contains a "spike" : triangles $t_{1}$ and $t_{2}$ belong to two regions. Restoration of the boundary (c) is performed via an edge flip. (d) In $3 \mathrm{D}$, tetrahedra belong to two regions. the restoration of the boundary (e) is performed by a 4-4 transformation (similar to the 3-2 transformation, with one more input tetrahedra).

\section{Results}

In this section, we present a comparative study of our method with those obtained with the AMIRA mesh generation software. This program allows to generate meshes from isosurfaces, surfaces or discrete data [1]. Our approach is used for the ElectroMagnetic (EM) field and thermic 3D numerical simulations [20]. EM field computation is carried out by solving the Maxwell equations with incomplete first order edge elements discretization. Thermal simulations are based on the resolution of the Pennes bio heat equation with nodal finite element. We performed a comparative study with a sphere of diameter $200 \mathrm{~mm}$ for which we know the analytical formulation for the for the calculation of temperature rise caused by heat convection [22]. With AMIRA, we generated two meshes $S_{1}$ and $S_{2}$, the first mesh is built from an isosurface and the second from the input voxels. The third mesh denoted $S_{3}$ is generated with our method from the same set of voxels.

Figure 9 shows quality histograms $\left(Q_{1}-Q_{4}\right)$ for the meshes $S_{1}, S_{2}, S_{3}$. For both criteria, the quality of tetrahedra obtained with our method is very superior to what we obtained with AMIRA. We measured the discretization error as the Haussdorf distance for $S_{2}$ and $\mathrm{S}_{3}$ using $S_{1}$ as the reference mesh. We choose $S_{1}$ because it was generated from an isosurface. The distance is $0.31 \%$ of the bounding box diagonal for $S_{2}$ and $0.17 \%$ for $S_{3}$. The error for our method is approximately twice smaller. Table 1 shows the average and the minimum value of the quality criteria $Q_{1}, Q_{4}$ for all the tetrahedra of $S_{1}, S_{2}$ and $S_{3}$. Lines $\epsilon_{\max }$ and $\epsilon_{r m s}$ give 

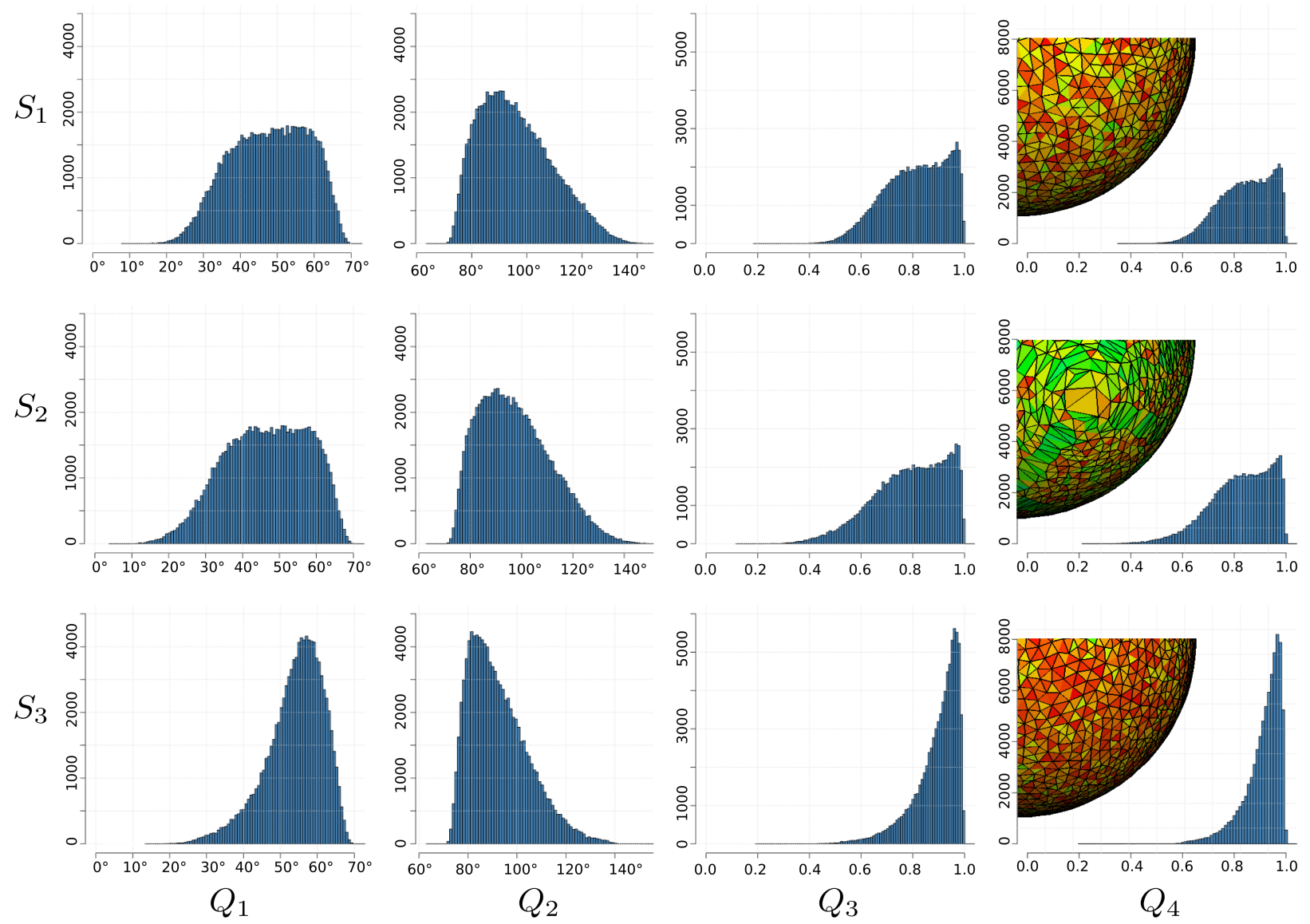

Fig. 9 Quality histograms for the meshes $S_{1}, S_{2}$ (generated with AMIRA) and $S_{3}$ constructed with our approach, for both criteria $Q_{1}$ - $Q_{4}$. $Q_{1}$ and $Q_{2}$ are the minimal and the maximal dihedral angle. $Q_{3}$ is the radius ratio. $Q_{4}$ is cited in [19]. The criterions $Q_{3}, Q_{4}$ are normalized between 0 and 1 , where 0 denotes a sliver and 1 a regular tetrahedron.

the maximal error and the average quadratic error between the result of the simulation and the exact analytical solution. These values show that our mesh $S_{3}$ brings an smaller error than $S_{2}$ does. However, this error is still greater than $S_{1}$. We interpret this result by the dominating role of the surface of the sphere for thermal simulation. For $S_{1}$, this surface is well defined by a surface mesh. The last two lines of the table show the number of iterations for the preconditioned conjugate gradient solver to compute the electromagnetic (1) and thermal (2) results.

For both simulations, the good quality of the tetrahedra provided by our method results in a significant reduction of the computation time.

The proposed algorithm has been tested with several discrete data. These algorithms were implemented in $\mathrm{C}++$ using the VTK library.

Figure 10 shows the results obtained on three different models. Armadillo (a), Stanford Bunny (b) and Venus body (c) are given as triangular meshes. For our approach, we voxelized these surface models. Histograms show the distributions of tetrahedra quality $Q_{2}$ for each mesh. Table 3 illustrates the effect of applying topological optimization on the tetrahedra quality. Table 2 shows the timings and quality measures for some results displayed in this paper. The results were obtained with a laptop computer running at $2.2 \mathrm{GHz}$, with $2 \mathrm{~GB}$ of RAM, except for the VHP model, which were processed on a SGI workstation due to memory requirements (The VHP model itself fits in more than 2GB with our data structure). We use our method to create a tetrahedral mesh from the Visible Human Project [2].

\section{Conclusion}

In this paper we have presented a very efficient and automatic method to construct the tetrahedral mesh from discrete data. Our approach produces a high quality tetrahedral mesh directly from discrete data using Centroidal Voronoi Diagrams. Our approach provides a robust mesh design tool for discrete datas that can accommodate requirements on the final budget of vertices and on the mesh gradation, for arbitrary domain complexity. Future work may include tetrahe- 


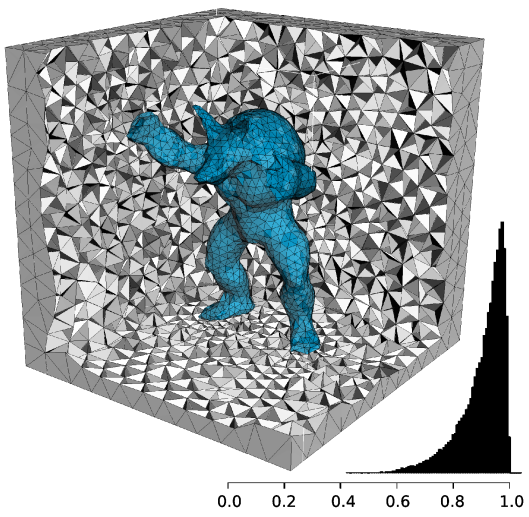

(a)

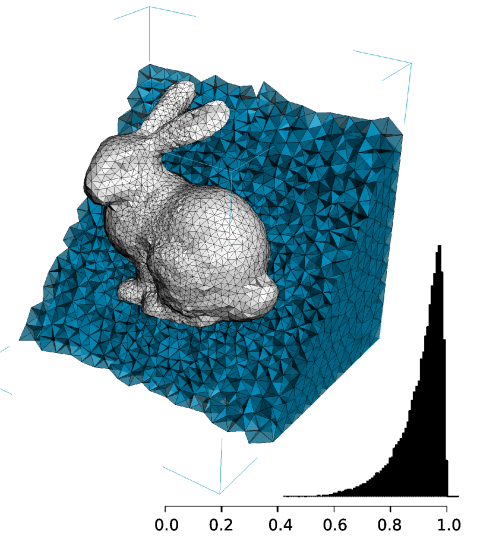

(b)

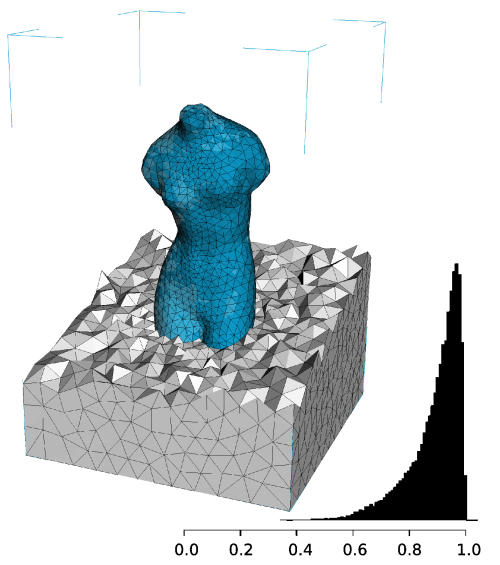

(c)

Fig. 10 This figure shows the results obtained on three different models. Armadillo (a), bunny (b) and venusbody (c) are given as triangular meshes. For our approach, we voxelized the input surface models. Histograms show the distributions of $Q_{4}$ for each tetrahedron in each meshes.

\begin{tabular}{|c|c|c|c|c|c|c|c|c|}
\hline \multirow[t]{2}{*}{ Model } & \multirow{2}{*}{$\begin{array}{c}\text { \# } \\
\text { tetrahedra }\end{array}$} & \multicolumn{3}{|c|}{ initial mesh } & \multicolumn{3}{|c|}{ after optimization } & \multirow{2}{*}{$\begin{array}{c}\text { minimum } \\
\text { dihedral angle }\left(^{\circ}\right)\end{array}$} \\
\hline & & $<6^{\circ}$ & $<12^{\circ}$ & $<18^{\circ}$ & $<6^{\circ}$ & $<12^{\circ}$ & $<18^{\circ}$ & \\
\hline armadillo & 55329 & 40 & 138 & 364 & 0 & 1 & 5 & $10.81^{\circ}$ \\
\hline bunny & 113409 & 99 & 358 & 654 & 0 & 1 & 8 & $11.54^{\circ}$ \\
\hline venusbody & 55103 & 33 & 113 & 226 & 0 & 5 & 13 & $10.17^{\circ}$ \\
\hline
\end{tabular}

Table 3 Evaluation of our optimization step for the quality of the generated meshes

\begin{tabular}{|c|c|c|c|}
\hline & $S_{1}$ & $S_{2}$ & $S_{3}$ \\
\hline$\overline{Q_{1}}$ & $51.97^{\circ}$ & $50.88^{\circ}$ & $56.32^{\circ}$ \\
\hline$\overline{Q_{4}}$ & 0.843 & 0.824 & 0.911 \\
\hline $\min \left(Q_{1}\right)$ & $13.41^{\circ}$ & $10.12^{\circ}$ & $16.31^{\circ}$ \\
\hline $\min \left(Q_{4}\right)$ & 0.362 & 0.282 & 0.376 \\
\hline$\epsilon_{\max }(1)$ & $0.035^{\circ} \mathrm{C}$ & $0.094^{\circ} \mathrm{C}$ & $0.047^{\circ} \mathrm{C}$ \\
\hline$\epsilon_{\text {rms }}(1)$ & 0.010 & 0.061 & 0.025 \\
\hline \# edges & 100875 & 102274 & 101027 \\
\hline \# iterations (1) & 171 & 205 & 121 \\
\hline \# iterations (2) & 5002 & 5004 & 4004 \\
\hline
\end{tabular}

Table 1 Quality of meshes and precision for FEM resolutions of thermic (1) and electromagnetic (2) simulations. Meshes $S_{1}$ and $S_{2}$ were obtained with AMIRA. $S_{3}$ was created with our approach.

\begin{tabular}{|c|c|c|c|c|}
\hline \# input & \# output & \multicolumn{3}{|c|}{ time (s) } \\
\cline { 3 - 5 } Voxels & Vertices & clustering & meshing & optimization \\
\hline $50^{3}$ & 500 & 2.42 & 0.44 & 0.72 \\
\hline $100^{3}$ & 4000 & 34.13 & 4.3 & 6.02 \\
\hline $200^{3}$ & 36000 & 271.96 & 36.11 & 42.26 \\
\hline
\end{tabular}

Table 2 Computation times with our approach

dral meshing on the human body and an octree approach to speed up the clustering step.

Acknowledgements The Visible Human Project [2] has provided the input images and driving force necessary to develop strategies and techniques to create numerically consistent, quantitative data representations of anatomical geometries. This work was supported in part by the BioRFMod project (ANR-06-JCJC-0124-01) and the Région Rhône Alpes Cluster 2 ISLE, PP3, subproject I3M: Imagerie Médicale et Modélisation Multiéchelles : du petit animal à l'Homme.

\section{References}

1. http://www.amiravis.com/

2. Ackerman, M.J.: From data to knowledge - the visible human project continues. In: HIC 2000: Proceedings, pp. 1-6 (2000)

3. Alliez, P., Cohen-Steiner, D., Yvinec, M., Desbrun, M.: Variational tetrahedral meshing. ACM Transactions on Graphics 24(3), 617-625 (2005)

4. Baker, T.J.: Element quality in tetrahedral meshes. In: Proceedings of the 7th International Conference On Finite Element Methods in Flow Problems, pp. 1018-1024 (1989)

5. Cheng, S.W., Dey, T.K., Edelsbrunner, H., Facello, M.A., Teng, S.H.: Sliver exudation. In: SCG '99: Proceedings on Computational geometry, pp. 1-13 (1999)

6. Choi, W.Y., Kwak, D.Y., Son, I.H., Im, Y.T.: Tetrahedral mesh generation based on advancing front technique and optimization scheme. International Journal for Numerical Methods in Engineering 58, 1857-1872 (2003)

7. Dardenne, J., S.Valette, Siauve, N., Prost, R.: Medial axis approximation with constrained centroidal voronoi diagrams on discrete data. In: Computer Graphics International, pp. 299-306 (2008)

8. Du Qiang and Wang Desheng: Tetrahedral mesh generation and optimization based on centroidal Voronoi tessellations. International Journal for Numerical Methods in Engineering, 56(9), 1355-1373 (2003).

9. Du, Q., Faber, V., Gunzburger, M. : Centroidal voronoi tesselations: applications and algorithms. SIAM Review, 41(4), 637-676 (1999). 
10. George, P.L., Seveno, E.: The advancing-front mesh generation method revisited. Journal for Numerical Methods in Engineering 37, 3605-3619 (1994)

11. Klingner, B.M., Shewchuk, J.R.: Agressive tetrahedral mesh improvement. In: Proceedings of the 16th International Meshing Roundtable, pp. 3-23 (2007)

12. Kovalevsky, V.A.: Finite topology as applied to image analysis. Comput. Vision Graph. Image Process. 46(2), 141-161 (1989)

13. Labelle, F., Shewchuk, J.R.: Isosurface stuffing: Fast tetrahedral meshes with good dihedral angles. In: Special issue on Proceedings of SIGGRAPH 2007, vol. 26, p. 57 (2007)

14. Marcum, D.L., Weatherill, N.P.: Shape reconstruction and volume grid generation using iterative point insertion and meshing for complex solids. AIAA Journal 33(9), 1619-1625 (1995)

15. Mitchell, S.A., Vavasis, S.A.: Quality mesh generation in higher dimensions. SIAM J. Comput. 29(4), 1334-1370 (2000)

16. Shewchuk, J.R.: Adaptive precision floating-point arithmetic and fast robust geometric predicates. Discrete and Computational Geometry 18, 305-363 (1997)

17. Shewchuk, J.R.: A condition guaranteeing the existence of higherdimensional constrained delaunay triangulations. In: SCG '98: Proceedings on Computational geometry, pp. 76-85 (1998)

18. Shewchuk, J.R.: Tetrahedral mesh generation by delaunay refinement. In: SCG '98: Proceedings on Computational geometry, pp. 86-95 (1998)

19. Shewchuk, J.R.: What is a good linear element? interpolation, conditioning, and quality measures. In: In Eleventh International Meshing Roundtable, pp. 115-126 (2002)

20. Siauve, N., Nicolas, L., Vollaire, C., Nicolas, A., Vasconcelos, J.: Optimization of $3 \mathrm{~d}$ sar distribution in local hyperthermia. IEEE Transactions on Magnetics 40(2), 1264-1267 (2004)

21. Bridson, R., Teran, J., Molino, N., Fedkiw, R., : Adaptive physics based tetrahedral mesh generation using level sets. Eng. with Comput. 21(1), 2-18 (2005)

22. Thiele, J., Golombeck, M., Dssel, O.: Thermal heating of human tissue induced by electromagnetic fields of magnetic resonance imaging. Biomedizinische Technik 47(2), 743-746 (2002)

23. Valette, S., Chassery, J.M., Prost, R.: Generic remeshing of $3 \mathrm{~d}$ triangular meshes with metric-dependent discrete voronoi diagrams. IEEE Trans Visu Comp Graph pp. 369-381 (2008)

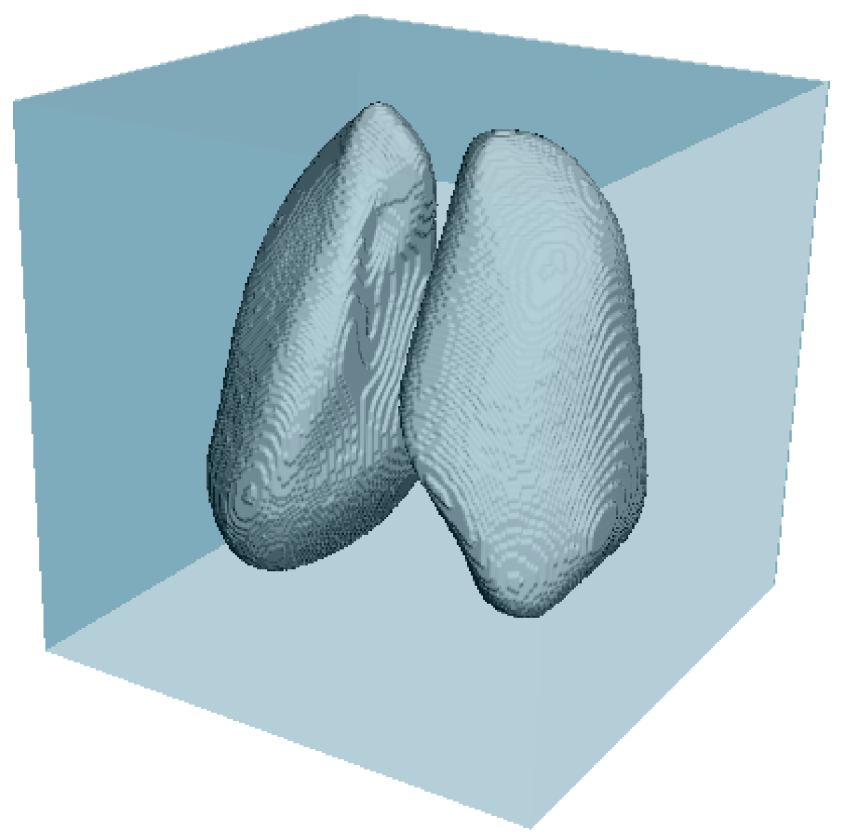

(a)

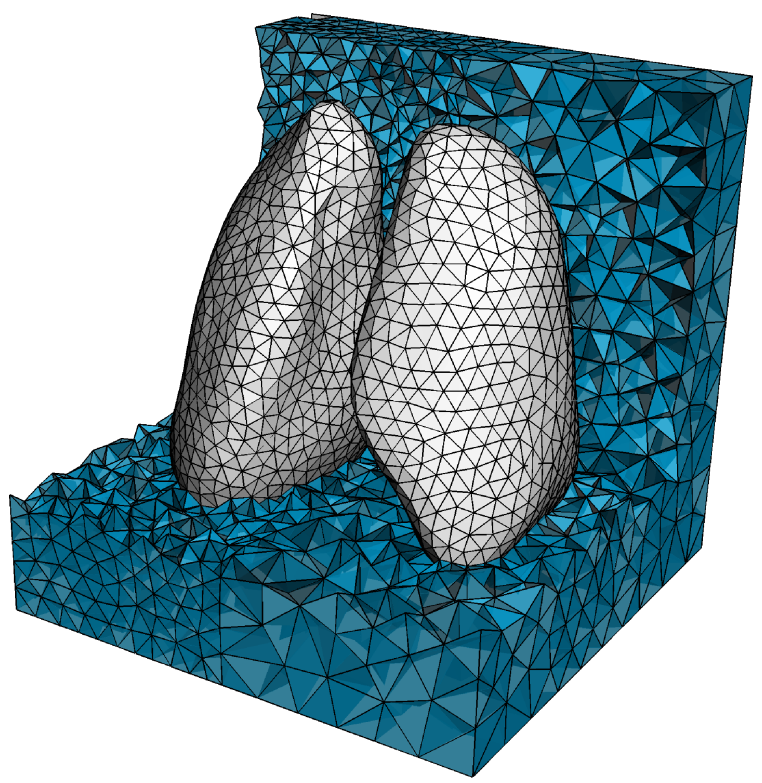

(b)

Fig. 11 (a), a volume representing human lungs. (b) is obtained with a density map which in one part possesses uniform weights (left lung) whereas in the other part respects certain complex regions (right lung). 